\title{
Decision heuristic or preference? \\ Attribute non-attendance in discrete choice problems
}

Short title: Decision heuristic or preference?

\section{Keywords}

Discrete choice experiment, attribute non-attendance, latent class, heuristic, thematic analysis

JEL C25, I10

Words 5439 (original submission: 4890)

Tables 5

Figures 3 


\section{INTRODUCTION}

Classical consumer theory requires individuals to consider and trade all attributes of a commodity when choosing between multi-attribute alternatives (de Palma et al. 1994, McIntosh \& Ryan 2002). This assumption is necessary to derive Hicksian welfare measures from discrete choice data (Dominitz \& Hung 2009, Zeppini 2015). The valuation of public goods like health care often relies on such welfare measures estimated from responses to stated preference surveys, such as discrete choice experiments (DCEs). In DCEs, respondents are presented with a series of hypothetical choice tasks. Each task consists of mutually exclusive multi-attribute alternatives (Lancaster 1966, Louviere et al. 2000, Ryan et al. 2008).

Empirical evidence suggests that individuals might not consider all commodity attributes when choosing between alternatives. This is known as attribute non-attendance or ANA (Scarpa et al. 2009). There are two main reasons for ANA: First, ANA occurs when individuals ignore attributes to simplify choice tasks. This explanation suggests that individuals have a limited cognitive capacity and respond to complex tasks with simplifying choice strategies called heuristics (Kahneman 1973, Malhotra 1982, Payne \& Bettman 2001). Second, ANA occurs when individuals ignore attributes they do not value. This explanation suggests respondents' preferences are reflected by ANA. ANA as a heuristic challenges discrete choice analysis, because such behaviour violates the assumption of continuous preferences (Campbell et al. 2008). In contrast, ignoring attributes that are unimportant is in line with the underlying assumptions of consumer theory (DeShazo \& Fermo 2004, Hensher 2006, Ryan et al. 2009).

In the DCE literature, several methods are used to account for ANA. Early studies focus on dominant choices that occur when respondents only consider one attribute and choose the alternative with the 'best' level. This results in a specific choice pattern that is identified by data inspection (Ryan \& Bate 2001, McIntosh \& Ryan 2002, Scott 2002, Sælensminde 2006). 
Other studies explore respondents' use of cut-off strategies in which attributes with a level above or below a threshold are considered (Swait \& Adamowicz 2001).

A broader definition of ANA is that respondents in a DCE with $k$ attributes either consider or ignore each attribute, which leads to $2^{k}$ possible ANA strategies (Hensher et al. 2005). These studies measure ANA using one of two approaches: stated ANA or inferred ANA. Stated ANA studies ask respondents to report the attributes they did not consider when making their choices (Puckett \& Hensher 2009, Scarpa et al. 2013). Inferred ANA studies use econometric models to identify ANA strategies probabilistically (Hess \& Rose 2007, Hole 2011, Lagarde 2013). Both approaches constrain individual parameters of attributes that were not considered to zero. Most stated and inferred ANA studies assume that ANA is a heuristic that respondents use to simplify choices, but ANA may be a preference. If ANA is a preference then methods that account for ANA, but assume it is a heuristic may result in distorted welfare measures.

Alemu et al. (2013) explore if ANA in their data may partially reflect references. The authors ask German tourists why they did not consider all attributes of presented Danish fishing sites. They find that between $13 \%$ and $84 \%$ of respondents who used ANA, ignored attributes that are not important to them. This is evidence of ANA as a preference. However, Alemu et al (2013) use stated ANA and respondents' ability to reflect on how they make their decisions has been suggested to be biased (Hess \& Hensher 2010; Nisbett \& Wilson 1977).

This is the first paper to assess if ANA is a heuristic or genuine preference in an inferred ANA study and the first study to explore this question within a health context. We assume that respondents' familiarity with the good being valued in the DCE simplifies choices. If ANA is mainly used as a heuristic, familiar respondents should be more likely to consider all attributes. We complement our empirical findings with a qualitative analysis of transcripts from discussion groups held prior to the DCE. This analysis is used to investigate why some 
respondents do not value all attributes. We also explore implications of assuming ANA is a heuristic or a preference for welfare analysis.

\section{EXPERIMENTAL DESIGN}

\subsection{Familiarity and ANA}

Several studies in the DCE literature (Neuman et al. 2010, Czajkowski et al. 2012, LaRiviere et al. 2014, Li et al. 2014) and contingent valuation literature (Boyle 1989, Cameron and Englin 1997) have explored the effect of experience and familiarity on respondents' preferences. Common explanations for this effect are that preferences may be incomplete, instable or constructed over the sequence of choices (Hoeffler \& Ariely 1999). Other studies find evidence that respondents prefer their familiar current situation (Salkeld et al. 2000, Ryan and Ubach 2003), which is known as status-quo bias or endowment effect.

In this paper, we assume that familiarity with the good being valued simplifies respondents' choices. Cognitive psychology research suggests that individuals form a mental model that is linked to expectations and preferences before making choices (Payne et al. 1999). This requires the dedication of cognitive effort. DCE respondents who are familiar with the good under valuation already have a mental model and therefore need to dedicate less cognitive effort to the completion of choice tasks. This is supported by studies that find familiarity to increases information access and information encoding (Koriat 1993, Metcalfe et al. 1993, Koriat \& Levy-Sadot 2001). Following this, we expect that familiar DCE respondents are less likely to use a decision heuristic to simplify choices than unfamiliar respondents. We also expect differences in ANA between familiar and unfamiliar respondents to be greater for attributes that respondents are more likely to be familiar with. 


\subsection{Data}

We use data from a DCE study about medical managers' preferences for support services offered by the National Clinical Assessment Service (NCAS). NCAS assists NHS organisations with performance concerns with clinicians. The study is reported in detail by Watson et al. (2012).

The DCE has five attributes, identified by Watson et al. (2012) from discussion groups with medical managers responsible for the management of clinicians. These attributes describe services that NCAS offered in 2010 or could potentially offer (Column 1-2 in Table I). From these attributes, 512 possible support packages can be formed. This number is reduced to 32 choice tasks using a D-efficient design with flat priors (Kuhfeld 2009). To avoid overburdening respondents, the choice tasks are split into three blocks ( $2 \times 11$ choices, 1 x 10 choices). An $11^{\text {th }}$ dummy choice set is added to the third block, such that all respondents face the same number of choices. This dummy choice set is excluded from the analysis. Each of the choice sets presents respondents with two alternative support services and an opt-out option. Both services are described as an insurance package with costs per whole time equivalent clinician in the organisation. Figure 1 presents an example choice set.

\section{[Figure 1]}

The questionnaire is mailed to all 1,875 NHS managers responsible for managing performance concerns in the United Kingdom who have not participated in the discussion groups. In this population, familiarity with NCAS varies for two reasons: First, not all organisations have experienced performance concerns. Second, NCAS services have been introduced at different times across the UK (England in 2001, Wales in 2003, Northern Ireland in 2005 and Scotland 
in 2008). ${ }^{1}$ Respondents' familiarity with NCAS is measured by asking them "Have you ever contacted NCAS about clinical performance concerns?" with the response options "Yes" or "No". Respondents are split into two groups based on their experience with NCAS. Respondents who previously contacted NCAS will have received at least some advice services about how to manage performance concerns with clinicians. We assume that experience with NCAS increases respondents' familiarity with the offered services, which are the attributes of the DCE. Given that ANA is attribute specific, we argue that this experience-based familiarity with services (attributes) is relevant to explain ANA in the DCE rather than a general familiarity with NCAS as an organisation.

\section{[Table I]}

Furthermore, respondents' familiarity varies across attributes. The attributes advice, facilitation and clinical assessment are services that accumulate with the performance concern's severity. Clinical assessments are only used in severe cases. By 2012, only $4.5 \%$ of organisations who contacted NCAS received clinical assessments (NCAS 2012). Behavioural assessments have not been offered by NCAS in 2010 (at the point of data collection). Hence, familiar respondents are more likely to have experienced advice and facilitation services from NCAS.

Across the familiar and unfamiliar respondent sub-groups, we do not expect respondents to differ in their familiarity with clinical or behavioural assessments, because these are rare and unavailable, respectively. We also expect respondents' sensitivity to the cost of services to be independent of their familiarity with NCAS. This is, because the marginal disutility of changes in the cost attribute represents NHS managers' general sensitivity to changes in the available budget due to increasing expenditure, while holding all remaining attributes constant.

\footnotetext{
${ }^{1}$ We explored the location of the NHS organisations as a measure of familiarity. However, given the small share of Welsh, Irish and Scottish NHS organisations in the sample, this approach is not feasible in our analysis.
} 
If familiarity is the main driver of ANA differences between the two groups of respondents, we should not find small differences in ANA for clinical assessment or the cost attributes (Column 3-4 in Table I).

\section{ANALYSIS}

\subsection{Econometric analysis}

We use a latent class logit to identify ANA strategies. This model assumes that the population can be divided into $C$ groups, and each group uses a different ANA strategy. The five attributes of the DCE result in $2^{5}=32$ possible combinations of attributes (ANA strategies) that respondents can consider when making their choices (e.g. one ANA strategy may be that only the first three attributes are considered, while the remaining two are ignored). The set of ANA strategies includes full attendance to all attributes and non-consideration of all attributes.

A utility function is formulated for each ANA strategy. We assume that the utility $U_{n j t}$ of respondent $n$ for alternative $j$ in choice situation $t$ depends on ANA strategy $c$, the vector of attributes $\boldsymbol{x}_{n j t}$ and an extreme value distributed random component $\varepsilon_{n j t}$ (McFadden 1974, Manski 1977). We define the utility function as:

$$
U_{n j t}\left(\boldsymbol{\delta}_{c}\right)=\left(\boldsymbol{\beta} \circ \boldsymbol{\delta}_{c}\right)^{\prime} \boldsymbol{x}_{n j t}+\varepsilon_{n j t}
$$

where $\boldsymbol{\beta}$ denotes a vector of the individual marginal utilities to be estimated. These marginal utilities are equal across classes. The vector $\boldsymbol{\delta}_{c}$ is multiplied element-by-element with $\boldsymbol{\beta}$ and contains only ones and zeroes. An ANA strategy is represented by a characteristic combination of the ones and zeros in $\boldsymbol{\delta}_{c}$ (Hess et al. 2013). Marginal utilities are constrained to be equal across classes to reduce the risk of confounding ANA with preference heterogeneity. If a categorical attribute is not considered, each corresponding parameter is constrained to zero 
(Scarpa et al. 2009). An alternative specific constant (asc) is included to control for status-quo effects.

The probability that respondent $n$ chooses alternative $j$, given ANA strategy $c$ is given by

$$
P_{n j t}\left(\boldsymbol{\delta}_{c}\right)=\frac{\exp \left[\left(\boldsymbol{\beta} \circ \boldsymbol{\delta}_{c}\right)^{\prime} \boldsymbol{x}_{n j t}\right]}{\sum_{j \in[1 ; 3]} \exp \left[\left(\boldsymbol{\beta} \circ \boldsymbol{\delta}_{c}\right)^{\prime} \boldsymbol{x}_{n j t}\right]}
$$

A priori, we do not know the ANA strategy used by each individual. Thus, the probability $\pi_{c}$ that a respondent uses ANA strategy $c$ is estimated using a multinomial logit defined as

$$
\pi_{c}=\frac{\exp \left(\theta_{c}\right)}{\sum_{c \in[1 ; \mathrm{C}]} \exp \left(\theta_{c}\right)}
$$

The parameters $\theta_{c}$ are estimated with one being constrained to zero for the identification of the model. Further details on the latent class logit are presented by Greene \& Hensher (2003) and Heckman \& Singer (1984). All models are estimated using python biogeme (Bierlaire 2003).

A conditional logit is used as reference model and the latent class model is specified in two steps (Figure 2). First, we estimate a model with all 32 ANA strategies. Second, we exclude ANA strategies with a probability smaller than $0.1 \% .{ }^{2}$ Reducing a general specification with 32 classes to a more parsimonious model eases the computational burden and reduces the risk of overfitting (Heckman \& Singer 1984). Furthermore, this approach is in line with the confirmatory nature of an ANA latent class model based on hypotheses about the existence of particular information processing strategies. This is in contrast to a latent class models used to explore preference heterogeneity, in which the number of classes is increased in a step-wise approach. The robustness of the model specification and identification has been explored in a

\footnotetext{
${ }^{2}$ Classes with a zero probability are not identified, because equation 3 is bound at zero. The latent class models with all 32 classes for familiar and unfamiliar respondents are available from the authors on request.
} 
simulation study and the results are available in Online Supplementary Information. ${ }^{3}$ The estimated models are compared based on the Akaike Information Criterion (AIC), the Bayesian Information Criterion (BIC) and the adjusted McFadden $\mathrm{R}^{2}\left(\bar{\rho}^{2}\right)$.

We compare the estimated probability $\pi_{n o \_A N A}$ of the full attendance class between the two groups of respondents to explore if ANA is a heuristic. If the probability that familiar respondents consider all attributes is smaller than the probability that unfamiliar respondents consider all attributes, we conclude that ANA mostly represents preferences.

We calculate the probability $\varpi_{k}$ of each attribute $k$ being ignored (ANA share) as the sum of the probabilities of all ANA strategies that do not consider attribute $k$. Standard errors of these estimates are obtained using the delta method and used to calculate confidence intervals (Oehlert 1992). We compare the ANA share for each attribute across familiar and unfamiliar respondents. The difference between $\varpi_{k}$ estimates across models cannot be tested statistically, because $\pi_{c}$ is not an element of a convex parameter space and the latent class models are not nested (Greene \& Hensher 2003). Distributions of conventional statistics like the likelihoodratio are unknown (McLachlan \& Peel 2000). Thus, we base our judgement on confidence intervals. If confidence intervals of parameters do not overlap, we conclude that they are significantly different. However, the converse is not always true (Schenker \& Gentleman 2001, Ryan \& Leadbetter 2002, Knol et al. 2011). We therefore calculate the distance between point estimates of ANA shares to explore differences intuitively. We hypothesise that these distances are larger for attributes with a different level of familiarity in both groups.

We also explore the number of attributes respondents consider on average. Respondents who consider more attributes experience a higher cognitive burden. We expect that if ANA is a

\footnotetext{
${ }^{3}$ We thank one anonymous reviewer for motivating us to explore our identification strategy in detail, which resulted in the Online Supplementary Information.
} 
heuristic, unfamiliar respondents will consider fewer attributes than familiar respondents. To explore this, the probability of not considering a specific number of attributes is calculated by summing the corresponding probabilities $\pi_{c}$ of ANA strategies.

\subsection{Qualitative analysis}

Watson et al. (2012) hold moderated face to face discussion groups with 23 senior NHS managers prior to the DCE to help develop the study questionnaire and derive attributes and levels. Participants are sampled from the same population as DCE respondents. Participants are presented with vignettes describing a scenario with a clinician about whom performance concerns have been raised and asked how their organisation would manage such a situation. We opportunistically use this qualitative data to investigate if some respondents do not value some DCE attributes. This provides insights into the validity of the quantitative findings. If ANA represents preferences, we expect some NHS managers to express non-valuation of such attributes. Conversely, if ANA is a heuristic, there should be no evidence of non-valuation of included attributes.

An inductive thematic approach is used to analyse the transcripts of the discussion groups to identify non-valuation expressed by NHS managers. Statements of non-valuation are those in which the participant states that the service is/was not valuable or useful to them and that they would not want to have that service. We do not specify words that are to be used by participants, but are guided by the context of the statement within the broader discussion group conversation. A topic that describes the content and context of the discussion is assigned to each of these statements and used to identify overarching themes that explain why some NHS managers do not value some services. The findings are validated independently by two researches and disagreements resolved by discussion. 


\subsection{Welfare implications}

ANA can affect WTP estimates (Campbell et al. 2008, Hess et al. 2013). If respondents ignore an attribute other than cost, WTP of these respondents for this attribute is zero. However, if respondents ignore the cost attribute, WTP becomes infinite. We explore the effect of assuming ANA is a heuristic or genuine preference using two alternative WTP measures. These two measures differ in their assumption about the marginal utility of ignored attributes (Hole et al. 2014). The true average marginal utility $\beta_{k}^{*}$ of an attribute $k$, is given by the weighted average of the marginal utility $\left(\beta_{k}\right)$ of respondents who consider the attribute and those who $\operatorname{don't}\left(\beta_{k}^{A N A}\right): \beta_{k}^{*}=\left(1-\varpi_{k}\right) \beta_{k}+\varpi_{k} \beta_{k}^{A N A}$ The weights are the corresponding probabilities of considering $\left(\varpi_{k}\right)$ or ignoring $\left(1-\varpi_{k}\right)$ the attribute.

Assuming ANA is a heuristic, and there is no preference heterogeneity, respondents who ignore attribute $k$ have a marginal utility of $\beta_{k}$, but disregard this valuation to simplify the choice task. Thus, WTP for attribute $k$ is:

$$
W T P_{k}^{\text {heur }}=\beta_{k} /-\beta_{\text {cost }} .
$$

If ANA expresses preferences then attributes that are ignored have a marginal utility of zero $\left(\beta_{k}^{A N A}=0\right)$. Thus, to calculate WTP for attribute $\mathrm{k}$, we weight the $\beta_{k}$ and $\beta_{\text {cost }}$ by the probability that attribute $k$ and cost attribute are considered:

$$
W T P_{k}^{p r e f}=\frac{\left(1-\varpi_{k}\right) \beta_{k}}{-\left(1-\varpi_{c o s t}\right) \beta_{\text {cost }}}
$$

These WTP estimates can be used to calculate the total WTP for an existing or potential support package $i$, calculated as

$$
W T P_{i}^{*}=\sum_{k \neq a s c} W T P_{k}^{\varphi} x_{i k}-W T P_{a s c}^{\varphi}
$$


where $x_{i k}$ denotes the level of attribute $k$ in the support package and $\varphi \in[$ heur,pref] denotes if ANA is assumed to be a heuristic or a preference. $W T P_{a s c}^{\varphi}$ is respondents' WTP for avoiding the involvement of NCAS.

We calculate total WTP for four hypothetical support packages to explore how different assumptions about ANA affect welfare conclusions (Table V). The four support packages are chosen to cover a spread of different attribute combinations and to increase in value $\left(W T P_{1}^{*}<\right.$ $\left.W T P_{2}^{*}<W T P_{3}^{*}<W T P_{4}^{*}\right)$. This allows us to explore how false assumptions about ANA may affect welfare conclusions. This is important, because welfare distortions due to wrong assumptions about ANA are not necessarily constant across support packages, if differences in heuristic WTP or preference WTP are not the same across attributes.

The structure of the analysis is summarised in Figure 2.

[Figure 2]

\section{RESULTS}

\subsection{Descriptive statistics and model comparison}

Four hundred fifty-one of the 1,875 questionnaires are returned (Response rate $=24 \%) .{ }^{4}$ Of the 451 respondents, $330(73 \%)$ are familiar and $113(25 \%)$ are unfamiliar with NCAS services. 8 (2\%) respondents are excluded from the sample due to missing data on familiarity with NCAS. This leaves in total 13,875 observations for the analysis (excluding missing values).

Table II presents the estimation results. Columns 2-5 report the conditional logit model estimates for familiar and unfamiliar respondents under the assumption of no ANA. Columns

\footnotetext{
${ }^{4}$ While this response rate is low, Watson et al. (2012) note that it is comparable to other stated preference studies with medical managers.
} 
6-9 report the latent class model estimates for both groups of respondents. The final latent class model considers 23 ANA strategies for familiar respondents and 15 for unfamiliar respondents.

A likelihood ratio test rejects a constant only model for each estimated model $(\mathrm{p}<0.001)$ and all estimated parameters are significantly different from zero $(\mathrm{p}<0.001)$. We find that accounting for ANA improves the model fit for both subgroups, and the improvement is larger for familiar respondents. All coefficients have the expected sign. The positive constant suggests that respondents prefer not to buy a support package and thus prefer to deal with performance concerns in-house. This is intuitive, because external services incur financial costs and coordination effort.

\section{[Table II]}

\subsection{Familiarity and ANA}

Table III presents the estimated probabilities that familiar and unfamiliar respondents ignore each attribute. If familiarity decreases respondents' cognitive load and ANA is a heuristic, then unfamiliar respondents should have higher rates of ANA. In contrast, we find that familiar respondents are less likely to consider all attributes (4.95\%) compared to unfamiliar respondents (16.91\%). This suggests that differences in ANA between the respondent groups are not driven by heuristics. We argue that these differences reflect preferences.

[Table III]

The advice attribute is ignored by $37.02 \%$ of familiar respondents compared to $12.40 \%$ of unfamiliar respondents. Similarly, the facilitation attribute is ignored by $52.19 \%$ of familiar respondents compared to $30.08 \%$ of unfamiliar respondents. The difference in the proportion of familiar and unfamiliar respondents who ignore the clinical assessment attribute is less than $1.00 \%$. This is consistent with our expectation, because few respondents who are familiar with 
NCAS will have experienced a clinical assessment. Behavioural assessment is ignored by $72.14 \%$ of familiar respondents and $49.26 \%$ of unfamiliar respondents. Although this is a relatively large difference, caution is needed in the interpretation, because of the large confidence intervals with a spread of $34.41 \%$ for familiar respondents and $81.38 \%$ for unfamiliar respondents.

Figure 3 presents the estimated probability of ignoring a specific number of attributes by familiarity. Familiar respondents have a higher probability of ignoring three or more attributes compared to unfamiliar respondents $(49.14 \%$ vs. $26.68 \%)$. Unfamiliar respondents are more likely to use ANA strategies in which zero to two attributes are ignored (73.22\% vs $50.86 \%)$. This means that familiar respondents are more likely to use ANA, to consider fewer attributes and therefore to process less information than unfamiliar respondents. If ANA is a heuristic we would expect to find the opposite. These findings support the evidence of the differences in ANA being due to preferences while making no judgement about the behavioural assessment attribute due to the large confidence intervals around attendance probabilities.

\section{[Figure 3]}

\subsection{Qualitative analysis}

The original study used moderated discussion groups with hypothetical vignette scenarios to explore, which aspects of support services that assist with performance concerns with clinicians are valued by NHS managers (Watson et al. 2012). The authors analysed the collected data using a thematic analysis and the results were used to identify the DCE attributes. The authors acknowledged that there is evidence suggesting that some NHS managers do not value all identified aspects of support service. In this paper we follow-up with this concern using the same data and a similar qualitative method to understand why some managers do not value all support services. This may provide insights into ANA as preferences. 
From the qualitative analysis of the discussion group transcripts, we identify 47 statements that reveal non-valuation of particular services offered by NCAS. These statements are of interest in the ANA context, because if some respondents do not value some services then this is a preference-based reason to ignore corresponding attributes in the DCE. Based on 16 topics that describe the context and content of these non-valuation statements, we find four latent overarching themes: (1) service formality; (2) potential consequences; (3) alternative providers; and (4) service quality.

\section{Theme 1 - Service formality}

Some NHS managers consider NCAS as formal and bureaucratic. These managers find that the standardised processes that NCAS use, cost them time and impose an unnecessary burden on assessed clinicians. One respondent reflects on previous experience with NCAS advice and states:

R1: "NCAS is seen to be, to me, more of a bureaucratic add-on that we sort of have a long discussion on the telephone."

This NHS manager is reluctant to involve NCAS based on previous experience with unbeneficial services. Thus, a non-valuation of such services seems likely. In the DCE, managers' general reluctance to involve NCAS is likely to be captured by the positive sign of the asc, but can also be reflected in respondents' non-valuation of particular attributes.

\section{Theme 2 - Consequences of involving NCS}

NHS managers also raise concern about the consequences of involving NCAS, because it might imply that further organisational resources would have to be spent on retraining or equipment. One participant states: 
R11: "If the outcome is a [clinician] needs retraining [one of our biggest problems] is who the heck does that and who funds it?"

This theme explains service non-valuation and shows why respondents are reluctant to purchase support services. In the DCE, this is reflected by the positive sign of the opt-out constant. ANA may be affected by theme 2, if the expected consequences of NCAS involvement exceed the expected gains.

\section{Theme 3 - Alternative providers}

Several managers report in-house experience with performance concern management and that they do not need external advice or facilitation. For the DCE, this may suggest that some NHS managers already had mechanisms in place to manage performance concerns. These respondents therefore have a genuine reason to ignore attributes in the DCE. When discussing a hypothetical doctor with alcohol problems one manager says:

$$
\text { R10: "We would just deal with that internally." }
$$

This implies that NHS institutions can provide some services themselves and are not willing to pay for this externally.

\section{Theme 4 - Service quality}

Some managers report previously unsuccessful NCAS consultations. Two participants state:

R1: "They send me a letter which I then have to sub-edit because they've got things wrong, and most of the correspondence is about getting this letter right, which then at the end of the day they did not sign. [...] So they've added nothing to my... to assist me."

R5: 'Some guidance, yeah, or to say 'this is what you need...' and it was sort of 'we're not really sure what you should do about that one', which wasn't really helpful." 
These statements indicate that some managers don't value advice based on their familiarity with the service. Respondents with similar experience might not be willing to pay for these services either. Additionally, some respondents explicitly express a non-valuation of particular services. With respect to advice, one manager stated

\section{R4: "I suppose I don't want them to advise on process."}

These qualitative findings suggest that there may be reasons (e.g. alternative services) for NHS managers to genuinely not value particular services offered by NCAS. ${ }^{5}$ This supports our quantitative findings that suggest ANA is mainly a preference in this study.

\subsection{Welfare implications}

Table IV presents $W T P^{\text {heur }}$ and $W T P^{\text {pref }}$ measures for all models. The conditional logit WTP values (columns 1, 4) do not account for ANA and show that familiar respondents are willing to pay more than unfamiliar respondents for all services except for facilitation in form of mediation. See Watson et al (2012) for more discussion of these results.

\section{[Table IV]}

$W T P^{\text {heur }}$ (columns 2 and 5) and WTPpref(columns 3 and 6) are either comparable or noticeably smaller than conditional logit WTP estimates. This means that not accounting for ANA may overestimate valuations. Furthermore, our qualitative analysis suggests that familiar respondents are more likely to ignore advice and facilitation attributes, because they do not value them. WTP $P^{\text {heur }}$ values do not reflect this, given that familiar and unfamiliar respondents have similar valuations. In contrast, $W T P^{\text {pref }}$ suggest that familiar respondents are willing to

\footnotetext{
5 The large share of statements concerned with advice is due to other cases being in comparison rare $(4.5 \%$ are clinical assessments). However, all services were discussed in the discussion groups.
} 
pay less for facilitation and advice than unfamiliar respondents. Thus, WT $P^{\text {pref }}$ estimates have a higher face-validity.

To explore welfare implications of wrong assumptions about ANA in more detail, we present total WTP for four hypothetical support packages in Table V.

\section{[Table V]}

The welfare effect of not accounting for ANA differs across support packages. If ANA is a heuristic, then the WTP from the conditional logit underestimates the value of services 1-3 for familiar respondents by $£ 12.41(£ 82.41-£ 70.00)$ to $£ 39.61(£ 55.72-£ 16.11)$ and for unfamiliar respondents by $£ 6.89$ ( $£ 80.08-£ 73.17)$ to $£ 25.22(£ 95.58-£ 70.36)$. If ANA represents preferences, the conditional logit results underestimate the WTP of familiar respondents for services 1 and 2 (£16.11 vs. £36.99 and £70.00 vs. £70.39), but overestimates WTP for services 3 and 4 ( $£ 83.61$ vs. $£ 77.35$ and $£ 158.12$ vs. $£ 116.24)$.

The range of potential welfare distortions due to ANA is larger for familiar respondents and smaller for unfamiliar respondents when relying on preference WTP rather than heuristic WTP. To quantify the overall welfare effect, we use the expected WTP defined as a weighted average between the WTP of familiar and unfamiliar respondents. We find the expected heuristic WTP to be, on average, larger by a factor of 1.18 (1.08 to 1.30$)$ than the expected preference WTP. Thus, assuming that ANA is a heuristic when it actually reflects preferences results in overestimated welfare effects.

\section{DISCUSSION}

This is the first study that explores the extent to which ANA in DCEs might represent preferences using inferred ANA. The DCE data is from a study about NHS managers' preferences for support services that assist with the management of performance concerns with 
clinicians. We find that accounting for ANA improves the model fit and that familiar respondents are less likely to consider all attributes than unfamiliar respondents. This is not in line with the hypothesis that ANA is a heuristic. We therefore conclude that differences in ANA between the groups are due to preferences. Our econometric results are supported by an opportunistic qualitative analysis of discussion group transcripts that shows some managers do not value all services due to their experience with NCAS.

We compare WTP estimated under two assumptions: ANA is a heuristic and ANA is a preference. We find that the assumption about respondents' reason to ignore attributes affects welfare estimates. We use WTP estimates to compare different hypothetical support packages and find that respondents' expected WTP is 1.08 to 1.30 times larger when ANA is assumed to be a heuristic rather than a preference. This may have important implications for the allocation of health care resources based on DCE studies. For example, in an economic evaluation, wrong assumptions about ANA can affect the estimated benefits and consequently the cost-benefit ratio. This may result in suboptimal recommendations about whether a policy should be implemented or not. Our analysis has some limitations. (1) ANA might be statistically confounded with preference heterogeneity (Hess et al. 2013, Hensher et al. 2013, Collins et al. 2013). While we do not consider differences in taste within the two groups, our findings suggest that there are confounding effects between ANA due to preferences and ANA due to other behavioural reasons. (2) we assume that familiarity decreases cognitive load. This might not hold in every situation (e.g. if best-practice and routine choices have been established). In this study we expect the assumption to hold, because performance concerns with clinicians are a rare event (NCAS 2012) and the DCE alternatives are formulated as generic insurance packages. (3) our opportunistic thematic analysis is limited because the discussion groups are not designed to gather information about decision rules. Future studies 
may therefore use process tracking techniques such as think-aloud to overcome these limitations (Ryan et al. 2009). (4) familiarity might be correlated with cognitive ability. However, given the relatively homogenous sample of highly educated NHS managers in an occupation with very similar responsibilities, we have no reason to believe that this is the case in our study. (5) our discussion distinguishes between ANA as preferences and ANA as heuristic. However, there might be other reasons for respondents to not attend to attributes, including protest behaviour or disbelief of selected attributes (Alemu et al. 2013, Meyerhoff et al. 2014). ANA for these reasons may explain non-attendance of the cost attribute. However, little is known about whether protest or disbelief behaviour results in ANA, or other behaviour such as serial non-participation and non-response (Roger et al. 2005). Future research could follow up with this concern by employing qualitative techniques such as think aloud protocols (Ryan et al. 2009).

Our findings have practical implications for DCE practitioners: studies of ANA and its causes need to distinguish between attributes that are generally not important to the majority of respondents and attributes that are ignored by only some respondents. Attributes that are generally not important should be identified and excluded at the design stage (based on discussion groups and pilots). This paper is concerned with attributes that are ignored by only a subset of respondents. Previous studies assume that these attributes are only ignored to simplify choices (ANA as heuristic). However, we show that this assumption results in misleading welfare estimates, if ANA is a preference. Future research should therefore proceed with caution when accounting for ANA and investigate why respondents do not consider attributes. This is important, because ANA and the reasons for it are likely to differ across applications and samples. For example, the NHS managers in the application of this paper are well educated and used to making complex decisions. Other respondents (e.g. with less education) may struggle more with similar choices (Sælensminde 2006). This should be 
investigated in future studies, because little is known about the link between respondents' characteristics and the use of heuristics in health DCEs. Given the limitations of econometric modelling and subjective reflections, think aloud methods (Ryan et al. 2009) and eye-tracking (Krucien et al. 2014, Balcombe et al. 2015) may provide more insight into causes of ANA in different applications, especially at the design and evaluation stage of DCE studies.

\section{REFERENCES}

Alemu, M.H., Mørkbak, M.R., Olsen, S.B. \& Jensen, C.L. 2013, "Attending to the Reasons for Attribute Non-attendance in Choice Experiments", Environmental and Resource Economics, vol. 54, no. 3, pp. 333-359.

Balcombe, K., Fraser, I. \& McSorley, E. 2015, "Visual ttention and attribute attendance in multi-attribute choice experiments", Journal of Applied Econometrics, vol. 30, no. 3, pp. 447467.

Bierlaire, M. 2003, "BIOGEME: A free package for the estimation of discrete choice models", Proceedings of the 3rd Swiss Transportation Research Conference, Ascona, Switzerland, March 19-21.

Boyle, K.J. 1989, "Commodity Specification and the Framing of Contingent-Valuation Questions", Land Economics, vol. 65k pp. 57-43.

Cameron, T.A. \& Englin, J. 1997, "Respondent Experience and Contingent Valuation of Environmental Goods", Journal of Environmental Economics and Management, vol. 33, pp. 296-313.

Campbell, D., Hutchinson, W.G. \& Scarpa, R. 2008, "Incorporating Discontinuous Preferences into the Analysis of Discrete Choice Experiments", Environmental and Resource Economics, vol. 41, no. 3, pp. 401-407.

Collins, A.T., Rose, J.M. \& Hensher, D.A. 2013, "Specification issues in a generalised random parameters attribute nonattendance model", Transportation Research Part B: Methodological, vol. 56, pp. 234-253.

Czajkowski, M., Hanley, N. \& LaRiviere, J. 2012, The Effect of Experience on Preference Uncertainty: Theory and Empirics of Public and Quasi-Public Goods, Stirling Economics Discussion Paper edn, University of Stirling.

de Palma, A., Myers, G.M. \& Papageorgiou, Y.Y. 1994, "Rational Choice Under an Imperfect Ability To Choose", American Economic Review, vol. 84, no. 3, pp. 419-440. 
DeShazo, J.R. \& Fermo, G. 2004, Implications of rationally-adaptive pre-choice behaviour for the design and estimation of choice models, Working Paper edn, School of Public Policy and Social Research, University of California, Los Angeles.

Dominitz, J. \& Hung, A.A. 2009, "Empirical models of discrete choice and belief updating in observational learning experiments", Journal of Economic Behavior \& Organization, vol. 69, no. 2, pp. 94-109.

Greene, W.H. \& Hensher, D.A. 2003, "A latent class model for discrete choice analysis: contrasts with mixed logit", Transportation Research Part B: Methodological, vol. 37, no. 8, pp. 681-698.

Hanley, N., Shogren, J. \& White, B. 2013, Introduction to Environmental Economics, 2nd edn, Oxford University Press, Oxford.

Heckman, J.J. \& Singer, B. 1984, "Econometric duration analysis", Journal of Econometrics, vol. 24 , no. 1-2, pp. 63-132.

Hensher, D.A. 2006, "Revealing Differences in Willingness to Pay due to the Dimensionality of Stated Choice Designs: An Initial Assessment", Environmental and Resource Economics, vol. 34 , no. 1 , pp. $7-44$.

Hensher, D.A., Collins, A.T. \& Greene, W.H. 2013, "Accounting for attribute non-attendance and common-metric aggregation in a probabilistic decision process mixed multinomial logit model: a warning on potential confounding", Transportation, vol. 40, no. 5, pp. 1003-1020.

Hensher, D.A., Rose, J.M. \& Greene, W.H. 2005, "The implications on willingness to pay of respondents ignoring specific attributes", Transportation, vol. 32, no. 3, pp. 203-222.

Hess, S. \& Hensher, D.A. 2010, "Using conditioning on observed choices to retrieve individual-specific attribute processing strategies", Transportation Research Part B: Methodological, vol. 44, no. 6, pp. 781-790.

Hess, S. \& Rose, J.M. 2007, "A latent class approach to modelling heterogeneous information process strategies in stated preference studies ", Presented at the Oslo Workshop on Valuation Methods in Transport Planning, Oslo, March 19-20.

Hess, S., Stathopoulos, A., Campbell, D., O’Neill, V. \& Caussade, S. 2013, "It's not that I don't care, I just don't care very much: confounding between attribute non-attendance and taste heterogeneity", Transportation, vol. 40, no. 3, pp. 583-607.

Hoeffler, S. \& Ariely, D. 1999, "Constructing Stable Preferences: A Look Into Dimensions of Experience and Their Impact on Preference Stability”, Journal of Consumer Psychology, vol. 8, no. 2, pp. 113-139.

Hole, A.R. 2011, "A discrete choice model with endogenous attribute attendance", Economics Letters, vol. 110, no. 3, pp. 203-205. 
Hole, A.R., Norman, R. \& Viney, R. 2014, "Response Patterns in Health State Valuation using Endogenous Attribute Attendance and Latent Class Analysis", Health Economics, DOI: 10.1002/hec.3134

Kahneman, D. (ed) 1973, Attention and Effort, Englewood Cliffs, Prentice-Hall.

Koriat, A. 1993, "How do we know that we know? The accessibility model of the feeling of knowing", Psychological review, vol. 100, no. 4, pp. 609-639.

Koriat, A. \& Levy-Sadot, R. 2001, "The combined contributions of the cue-familiarity heuristic and accessibility heuristic to feeling of knowing", Journal of Experimental Psychology: Learning, Memory, and Cognition, vol. 27, no. 1, pp. 34-53.

Krucien, N., Ryan, M. \& Hermens, F. 2014, "Using eye-tracking to inform the decisionmaking process in discrete choice experiments", Health Economists' Study Group (HESG) MeetingGlasgow Caledonian University, Glasgow, 23-25 June.

Kuhfeld, W.F. 2010. "Marketing Research Methods in SAS: Experimental Design, Choice, Conjoint, and Graphical Techniques, SAS Institute, Cary.

Lagarde, M. 2013, "Investigating attribute non-attendance and it's consequences in choice experiments with latent class models", Health Economics, vol. 22, no. 5, pp. 554-567.

Lancaster, K.J. 1966, "A New Approach to Consumer Theory", Journal of Political Economy, vol. 74, no. 2, pp. 132-157.

LaRiviere, J., Czajkowski, M., Hanley, N., Aanesen, M., Falk-Petersen, J. \& Tinch, D. 2014, "The value of familiarity: Effects of knowledge and objective Signals on willingness to pay for a public good", Journal of Environmental Economics and Management, vol. 68, pp. 376389.

Li, X., Boyle, K.J., Holmes, T.P. \& LaRouche, G.O. 2014, “The effect of on-site forest experience on stated preferences for low-impact timber harvesting programs", Journal of Forest Economics, vol. 20, no. 4, pp. 348-362.

Louviere, J.J., Hensher, D.A. \& Swait, J. 2000, Stated Choice Methods - Analysis and Applications, Cambridge University Press, Cambridge.

Malhotra, N.K. 1982, "Information Load and Consumer Decision Making", Journal of Consumer Research, vol. 8, no. 4, pp. 419-430.

Manski, C.F. 1977, "The structure of random utility models", Theory and Decision, vol. 8, no. 3, pp. 229-254.

McFadden, D. 1974, "Conditional logit analysis of qualitative choice behaviour" in Frontiers in econometrics, ed. P. Zarembka, Academic Press, New York, pp. 105-142.

McIntosh, E. \& Ryan, M. 2002, "Using discrete choice experiments to derive welfare estimates for the provision of elective surgery: Implications of discontinuous preferences", Journal of Economic Psychology, vol. 23, no. 3, pp. 367-382. 
McLachlan, G. \& Peel, D. 2000, Finite Mixture Models, Wiley-Blackwell, New York.

Metcalfe, J., Schwartz, B.L. \& Joaquim, S.G. 1993, "The cue-familiarity heuristic in metacognition.", Journal of Experimental Psychology: Learning, Memory, and Cognition, vol. 19 , no. 4 , pp. 851-861.

Meyerhoff, J., Mørbak, M.R. \& Olsen, S.B. 2014,“A Meta-study Investigating the Sources of Protest Behaviour in Stated Preference Surveys", Environmental and Resource Economics, vol. 58, no. 1, pp. 35-57.

NCAS 2012, Casework Activity Report (2011/12).

Neuman, T., Neuman, E. \& Neuman, S. 2010, "Explorations of the effect of experience on preferences for a health-care service", The Journal of Socio-Economics, vol. 39, no. 3, pp. 407-419.

Nisbett, R.E. \& Wilson, T.D. 1977, "Telling More Than We Can Know: Verbal Reports On Mental Processes", Psychological Review, vol. 84, no. 3, pp. 231-259.

Oehlert, G.W. 1992, "A Note on the Delta Method", The American Statistician, vol. 46, no. 1, pp. 27-29.

Payne, J.W. \& Bettman, J.R. 2001, "Preference Choice and Adaptive Strategy Use" in Bounded Rationality: The Adaptive Toolbox - Dahlem Workshop Reports, eds. G. Gigerenzer \& R. Selten, The MIT Press, Cambridge, pp. 103-121.

Payne, J.W., Bettman, J.R. \& Schkade, D.A. 1999, "Measuring Constructed Preferences: Towards a Building Code", Journal of Risk and Uncertainty, vol. 19, no. 1-3, pp. 243-270.

Puckett, S.M. \& Hensher, D.A. 2009, "Revealing the extent of process heterogeneity in choice analysis: An empirical assessment", Transportation Research Part A: Policy and Practice, vol. 43, no. 2, pp. 117-126.

Ritchie, J., Lewis, J., McNaughton Nicholls, C. \& Ormston, R. (eds) 2003, Qualitative Reseach Practice: A Guide for Social Science Students and Researchers, SAGE publications, London.

Rose, J.M. \& Bliemer, M.M.C.J. 2007, "Stated preference experimental design strategies" in Handbook of Transport Modelling, eds. D.A. Hensher \& K.J. Butto, 2nd edn, Elsevier Science, Oxford, pp. 151-180.

Roger, H., Massey, D.M., Adamovicz. W.L. 2005, "Serial Nonparticipation in Repeated Discrete Choice Models", American Journal of Agricultural Economics, vol. 87, no. 4, pp. 1061-1076.

Ryan, M. \& Bate, A. 2001, "Testing the assumptions of rationality, continuity and symmetry when applying discrete choice experiments in health care", Applied Economics Letters, vol. 8, no. 1, pp. 59-63. 
Ryan, M., Gerard, K. \& Amaya-Amaya, M. 2008, "Discrete Choice Experiments in a Nutshell" in Using Discrete Choice Experiments to Value Health and Health Care, eds. M. Ryan, K. Gerard \& M. Amaya-Amaya, Springer, Dordrecht, pp. 13-46.

Ryan, G.W. \& Leadbetter, S.D. 2002, „On The Misuse Of Confidence Intervals For Two Means In Testing For The Significance Of The Difference Between The Means, Journal of Modern Applied Statistical Methods, vol. 1, no. 2, pp. 473-478.

Ryan, M. \& San Miguel, F. 2003, "Revisiting the axiom of completeness in health care", Health Economics, vol. 12, no. 4, pp. 295-307.

Ryan M. \& Ubach C. 2003, "Testing for an experience endowment effect within choice experiments", Applied Economic Letters, vol.10, pp. 407-410.

Ryan, M., Watson, V. \& Entwistle, V. 2009, "Rationalising the 'irrational': a think aloud study of discrete choice experiment responses", Health Economics, vol. 18, no. 3, pp. 321336.

Salkeld G, Ryan M. \& Short L. 2000, "The veil of experience: do consumers prefer what they know best?”, Health Economic, vol. 9, pp. 267-270.

Sælensminde, K. 2006, "Causes and consequences of lexicographic choices in stated choice studies", Ecological Economics, vol. 59, no. 3, pp. 331-340.

Scarpa, R., Gilbride, T.J., Campbell, D. \& Hensher, D.A. 2009, "Modelling attribute nonattendance in choice experiments for rural landscape valuation", European Review of Agricultural Economics, vol. 36, no. 2, pp. 151-174.

Scarpa, R., Zanoli, R., Bruschi, V. \& Naspetti, S. 2013, "Inferred and Stated Attribute Nonattendance in Food Choice Experiments", American Journal of Agricultural Economics, vol. 95, no. 1, pp. 165-180.

Schenker, N. \& Gentleman J.F. 2001, “On Judging the Significance of Differences by Examining the Overlap Between Confidence Intervals", The American Statistician, vol. 55, No. 3, pp.182-186.

Scott, A. 2002, "Identifying and analysing dominant preferences in discrete choice experiments: An application in health care", Journal of Economic Psychology, vol. 23, no. 3, pp. 383-398.

Swait, J. \& Adamowicz, W. 2001, "The Influence of Task Complexity on Consumer Choice: A Latent Class Model of Decision Strategy Switching", Journal of Consumer Research, vol. 28, no. 1, pp. 135-148.

Watson, V., Sussex, J., Ryan, M. \& Ebenezer, T. 2012, "Managing poorly performing clinicians: Health care providers' willingness to pay for independent help", Health Policy, vol. 104, no. 3 .

Zeppini, P. 2015, "A discrete choice model of transitions to sustainable technologies", Journal of Economic Behavior \& Organization, vol. 112, no. 0, pp. 187-203. 
Table I Attributes and levels of the DCE

\begin{tabular}{|c|c|c|c|}
\hline & & \multicolumn{2}{|c|}{$\begin{array}{c}\text { Expected ANA share } \\
\text { (assuming ANA is a heuristic) }\end{array}$} \\
\hline Attributes & Attribute levels and corresponding parameters & Unfamiliar respondents & Familiar respondents \\
\hline Advice & $\begin{array}{l}\text { (1) no advice } \\
\text { (2) web based }\left(\beta_{a d_{\_} \text {web }}\right) \\
\text { (3) web based + telephone }\left(\beta_{\text {ad_phone }}\right) \\
\text { (4) web based + telephone + written summary }\left(\beta_{\text {ad_full }}\right)\end{array}$ & High & Low \\
\hline Facilitation & $\begin{array}{l}\text { (1) no facilitation } \\
\text { (2) facilitation }\left(\beta_{\text {fa_mediation }}\right) \\
\text { (3) facilitation + action plan }\left(\beta_{f a \_p l a n}\right) \\
\text { (4) facilitation + action plan + monitoring the implementation }\left(\beta_{f a} \text { full }\right)\end{array}$ & High & Low \\
\hline Behavioural assessment & $\begin{array}{l}\text { (1) no behavioural assessment } \\
\text { (2) availability of behavioural assessment ( } \beta_{\text {be_assess }} \text { ) }\end{array}$ & \multicolumn{2}{|c|}{ Small difference only } \\
\hline Clinical assessment & $\begin{array}{l}\text { (1) no clinical assessment } \\
\text { (2) limited assessment }\left(\beta_{\text {cl_limited }}\right) \\
\text { (3) full assessment }\left(\beta_{\text {cl_n__orga }}\right) \\
\text { (4) full assessment + assessment of organisational factors }\left(\beta_{\text {cl_full }}\right)\end{array}$ & \multicolumn{2}{|c|}{ Small difference only } \\
\hline Cost & 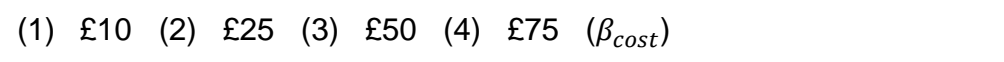 & \multicolumn{2}{|c|}{ Small difference only } \\
\hline
\end{tabular}


Table II Estimated marginal utilities and information criteria a

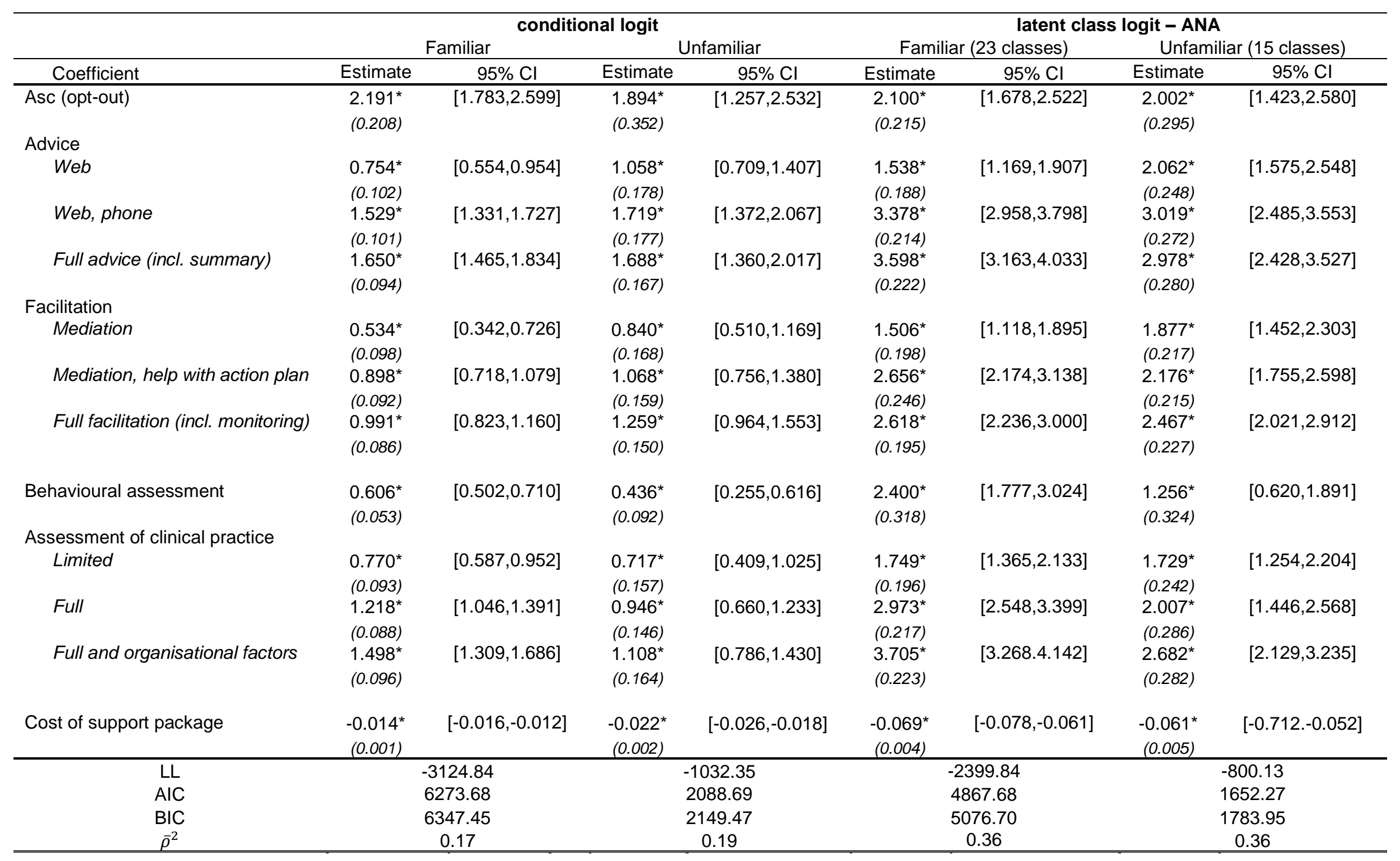

${ }^{a}$ Standard errors in parentheses * $p$-value $<0.001$ 
Table III Estimated probabilities of ANA by attribute, based on the latent class model a

\begin{tabular}{lrccc|c}
\hline & \multicolumn{2}{c}{ Familiar } & \multicolumn{2}{c}{ Unfamiliar } & \multirow{2}{*}{ ifference } \\
& Prob. & $95 \% \mathrm{Cl}$ b & Prob. & $95 \% \mathrm{Cl}^{\mathrm{c}}$ & ( \\
\hline Probability of considering all attributes & $4.95 \%$ & {$[-1.62 \%, 11.51 \%]$} & $16.91 \%$ & {$[5.12 \%, 28.70 \%]$} & $11.96 \%$ \\
\hline Probability of ignoring & & & & & \\
\hline Advice & $37.02 \%$ & {$[26.41 \%, 47.63 \%]$} & $12.40 \%$ & {$[2.10 \%, 22.70 \%]$} & $24.62 \%$ \\
Facilitation & $52.19 \%$ & {$[41.65 \%, 62.74 \%]$} & $30.08 \%$ & {$[18.87 \%, 41.30 \%]$} & $22.11 \%$ \\
Behavioural assessment & $72.14 \%$ & {$[47.32 \%, 96.96 \%]$} & $49.26 \%$ & {$[9.17 \%, 89.35 \%]$} & $22.88 \%$ \\
Clinical assessment & $34.49 \%$ & {$[24.26 \%, 44.72 \%]$} & $35.39 \%$ & {$[15.35 \%, 55.43 \%]$} & $0.90 \%$ \\
Cost of support package & $49.67 \%$ & {$[37.80 \%, 61.53 \%]$} & $40.60 \%$ & {$[26.12 \%, 55.03 \%]$} & $9.07 \%$ \\
\hline \hline
\end{tabular}

a Given the difficulty in interpreting $\theta_{c}$ parameters in equation 3 and corresponding inference directly, we only present the resulting ANA probabilities. However, the full estimates of the class membership model are available from the authors on request.

${ }^{b}$ confidence intervals are obtained using Delta method 
Table IV Estimated WTP for the different attributes based on the conditional logit and the latent class model

\begin{tabular}{|c|c|c|c|c|c|c|}
\hline \multirow[b]{3}{*}{ Coefficient } & \multicolumn{3}{|c|}{ Familiar } & \multicolumn{3}{|c|}{ Unfamiliar } \\
\hline & \multirow{2}{*}{$\begin{array}{c}\text { Conditional logit } \\
W T P^{\mathrm{a}} \\
\end{array}$} & \multicolumn{2}{|c|}{ Latent class model } & \multirow{2}{*}{$\frac{\text { Conditional logit }}{W T P \text { a }}$} & \multicolumn{2}{|c|}{ Latent class model } \\
\hline & & WTPheur a & WTP pref b & & WTP heur a & WTP $P^{\text {pref } \mathrm{b}}$ \\
\hline Asc (opt-out) & $£ 152.08^{*}$ & $£ 30.45^{\star}$ & $£ 60.50^{*}$ & $£ 86.09^{*}$ & $£ 32.57^{*}$ & $£ 54.38^{*}$ \\
\hline \multicolumn{7}{|l|}{ Advice } \\
\hline Web & $£ 52.36^{*}$ & $£ 22.30^{*}$ & $£ 27.90^{*}$ & $£ 48.09^{*}$ & $£ 33.54^{*}$ & $£ 49.46^{*}$ \\
\hline Web, phone & $£ 106.25^{*}$ & $£ 48.99^{*}$ & $£ 61.30^{*}$ & $£ 78.14^{*}$ & $£ 49.11^{*}$ & $£ 72.43^{*}$ \\
\hline Full advice (incl. summary) & $£ 114.58^{*}$ & $£ 52.18^{*}$ & $£ 65.29^{*}$ & $£ 76.73^{*}$ & $£ 48.44^{*}$ & $£ 71.44^{*}$ \\
\hline \multicolumn{7}{|l|}{ Facilitation } \\
\hline Mediation & $£ 37.08^{*}$ & $£ 21.84^{*}$ & $£ 20.75^{*}$ & $£ 38.18^{*}$ & $£ 30.54^{*}$ & $£ 35.95^{*}$ \\
\hline Mediation, help with action plan & $£ 62.36^{*}$ & $£ 38.51^{*}$ & $£ 36.58^{*}$ & $£ 48.55^{\star}$ & $£ 35.41^{*}$ & $£ 41.68^{*}$ \\
\hline Full facilitation (incl. monitoring) & $£ 68.82^{*}$ & $£ 37.96^{*}$ & $£ 36.06^{*}$ & $£ 57.23^{*}$ & $£ 40.13^{*}$ & $£ 47.24^{*}$ \\
\hline Behavioural assessment & $£ 42.08^{*}$ & $£ 34.80^{*}$ & $£ 19.27^{*}$ & $£ 19.82^{*}$ & $£ 20.43^{*}$ & $£ 17.45^{\star}$ \\
\hline \multicolumn{7}{|l|}{ Assessment of clinical practice } \\
\hline Limited & $£ 53.47^{*}$ & $£ 25.36^{*}$ & $£ 33.01^{*}$ & $£ 32.59^{\star}$ & $£ 28.13^{*}$ & $£ 30.60^{*}$ \\
\hline Full & $£ 84.72^{*}$ & $£ 43.12^{*}$ & $£ 56.12^{*}$ & $£ 43.00 *$ & $£ 32.66^{*}$ & $£ 35.52^{*}$ \\
\hline Full and organisational factors & $£ 104.17^{*}$ & $£ 53.73^{*}$ & $£ 69.93^{*}$ & $£ 50.36^{*}$ & $£ 43.64^{*}$ & $£ 47.47^{*}$ \\
\hline
\end{tabular}

a based on equation (4) ${ }^{b}$ based on equation (5)* ${ }^{*}$-value $<0.001$ 
Table V Total WTP for different potential support packages offered by NCAS

\begin{tabular}{|c|c|c|c|c|c|}
\hline & & \multicolumn{4}{|c|}{ Support packages } \\
\hline & & (1) & (2) & (3) & (4) \\
\hline & Web & $x$ & & $x$ & \\
\hline \multirow[t]{3}{*}{ Advice } & Web, phone & & $x$ & & \\
\hline & Full advice (incl. summary) & & & & $x$ \\
\hline & Mediation & & & $x$ & \\
\hline \multirow[t]{2}{*}{ Facilitation } & Mediation, help with action plan & $x$ & $x$ & & \\
\hline & Full facilitation (incl. monitoring) & & & & $x$ \\
\hline \multicolumn{2}{|c|}{ Behavioural assessment } & & & $x$ & $x$ \\
\hline & Limited & $x$ & $x$ & & \\
\hline \multirow[t]{2}{*}{ Clinical assessment } & Full & & & & $x$ \\
\hline & Full and organisational factors & & & $x$ & \\
\hline \multicolumn{6}{|l|}{ Conditional logit } \\
\hline \multicolumn{2}{|l|}{ Familiar respondents } & $£ 16.11$ & $£ 70.00$ & $£ 83.61$ & $£ 158.12$ \\
\hline \multicolumn{2}{|l|}{ Unfamiliar respondents } & $£ 43.14$ & $£ 73.19$ & $£ 70.36$ & $£ 110.69$ \\
\hline \multicolumn{2}{|c|}{ Familiar and unfamiliar respondents (weighted) } & $£ 23.00$ & $£ 70.81$ & $£ 80.23$ & $£ 146.02$ \\
\hline \multicolumn{6}{|l|}{ Latent class model } \\
\hline \multicolumn{6}{|l|}{ WTP $P^{\text {heur }}$} \\
\hline \multicolumn{2}{|l|}{ Familiar respondents } & $£ 55.72$ & $£ 82.41$ & $£ 102.22$ & $£ 137.61$ \\
\hline \multicolumn{2}{|c|}{ Unfamiliar respondents } & $£ 64.51$ & $£ 80.08$ & $£ 95.58$ & $£ 109.09$ \\
\hline \multicolumn{2}{|c|}{ Expected WTP of familiar and unfamiliar respondents } & $£ 57.96$ & $£ 81.82$ & $£ 100.53$ & $£ 130.34$ \\
\hline \multicolumn{2}{|l|}{ Familiar respondents } & $£ 36.99$ & $£ 70.39$ & $£ 77.35$ & $£ 116.24$ \\
\hline \multicolumn{2}{|c|}{ Unfamiliar respondents } & $£ 67.36$ & $£ 90.33$ & $£ 95.95$ & $£ 117.27$ \\
\hline \multicolumn{2}{|c|}{ Expected WTP of familiar and unfamiliar respondents } & $£ 44.74$ & $£ 75.48$ & $£ 82.09$ & $£ 116.50$ \\
\hline
\end{tabular}

${ }^{\mathrm{a} X}$ indicates available services

${ }^{\mathrm{b}}$ WTP of familiar and unfamiliar weighted with the share of each type of respondents in the sample 
Figure 1 Example choice set

\begin{tabular}{|c|c|c|c|}
\hline & Package $\mathbf{A}$ & Package B & No package \\
\hline 1. Advice & $\begin{array}{c}\text { d. Web-based resource + } \\
\text { telephone advice + written } \\
\text { confirmation }\end{array}$ & $\begin{array}{c}\text { c. Web-based resource + } \\
\text { telephone advice }\end{array}$ & No advice \\
\hline 2. Facilitation & $\begin{array}{c}\text { d. Facilitation/mediation }+ \text { help } \\
\text { with action plans and placements } \\
+ \text { monitoring of progress }\end{array}$ & b. Facilitation/mediation & No facilitation \\
\hline $\begin{array}{l}\text { 3. Behavioural \& health } \\
\text { assessment }\end{array}$ & a. Not available & b. Available & Not available \\
\hline $\begin{array}{l}\text { 4. Clinical assessment \& } \\
\text { organisational assessment }\end{array}$ & b. Limited clinical assessment & a. None & None \\
\hline 5. Annual cost/wte & $£ 75$ & $£ 50$ & $£ 0$ \\
\hline $\begin{array}{l}\text { Please tick one box and turn } \\
\text { to the next page. }\end{array}$ & Package A & Package B & $\begin{array}{l}\text { I would recommend no } \\
\text { package }\end{array}$ \\
\hline
\end{tabular}


Figure 2 Structure of the analysis

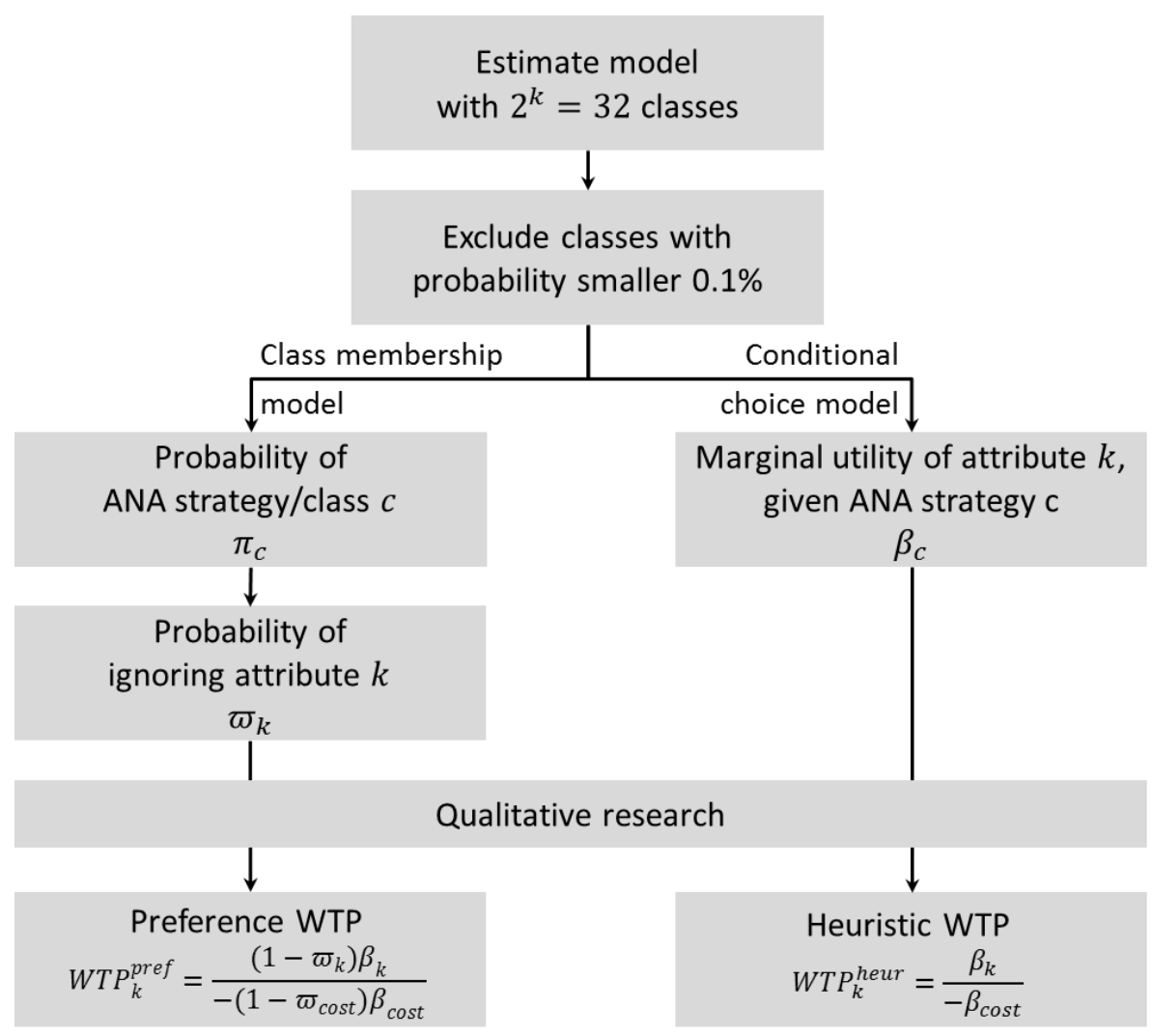


Figure 3 Estimated probability of ignoring a specific number of attributes

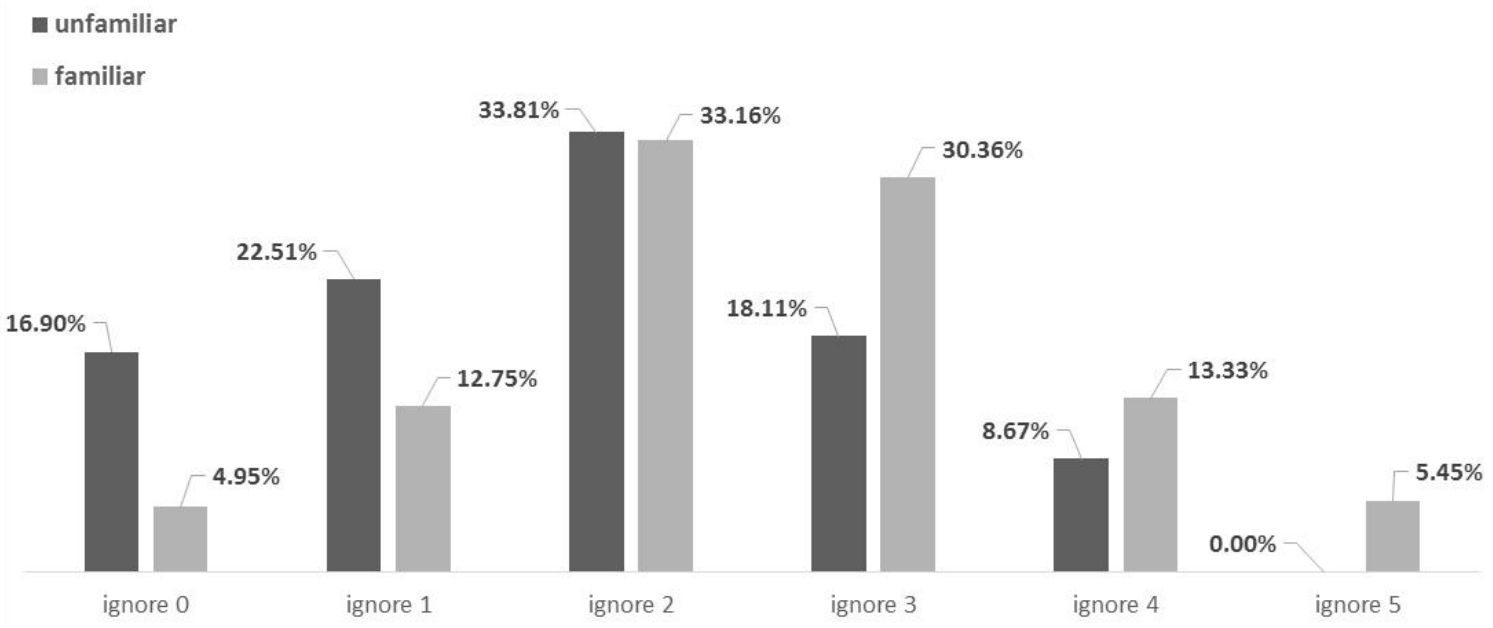

